\title{
The Clinical Use of Multi-modal Resources (2D/3D/Statistics) for Robot Assisted Functional Neurosurgery
}

\author{
Alim-Louis Benabid ${ }^{1}$, Dominque Hoffmann ${ }^{1}$, Luc Court ${ }^{1}$, Vincent Robert ${ }^{2}$, \\ Sébastien Burtin ${ }^{2}$,Patrick Pittet ${ }^{2}$, Jörg Fischer ${ }^{3}$ \\ ${ }^{1}$ Service de NeuroChirurgie, Centre Hospitalier Universitaire, Grenoble, France \\ ${ }^{2}$ Integrated Surgical Systems SA, Bron, France, ${ }^{3}$ IVS Solutions AG, Chemnitz, Germany
}

\begin{abstract}
This paper presents specific techniques and their implementation to integrate in the planing of functional neurosurgery all the available resources (3D preoperative examinations, intra-operative $2 \mathrm{D}$ radiographic X-rays, statistical database and atlases).

Keywords. Functional Neurosurgery - Image fusion - Co-Registration - Statistical Data - Stereotactic Robot.
\end{abstract}

\section{Introduction}

We present in this paper different methods to combine all the available information for planning of stereotactic functional neurosurgery and their implementation within a planning software $\left(V_{0 X i m}{ }^{\mathrm{TM}}\right.$, IVS Solutions AG) . This software is used to drive a stereotactic robot (NeuroMate ${ }^{\mathrm{TM}}$, ISS SA) for an optimal targeting of the planned trajectory[2][3].

\section{Co-registration and Fusion}

Co-registration of two modalities consists in the computation of the rigid transformation matrix ${ }^{\mathrm{im} 2} \mathrm{~T}_{\mathrm{im} 1}$ used to convert coordinates expressed in the first image referential in coordinates expressed in the second image referential. After co-registration of two modalities, it is possible to display a point defined on one modality on the other modality and vice-versa. Stereotactic trajectories can also been displayed on both modalities.

Fusion consists in the construction of a new hybrid data set from co-registred exams. Fusion allows a very synthetic management of multi-modalities. For stereotactic applications, Fusion does not bring any additional information to co-registered modalities.

\footnotetext{
${ }^{1}$ This work has been granted by the French Government

W. Niessen and M. Viergever (Eds.): MICCAI 2001, LNCS 2208, pp. 1421-1423, 2001.

(C) Springer-Verlag Berlin Heidelberg 2001
} 


\subsection{Fusion of Tri-dimensional Data Sets}

Different methods have been implemented for fusion of $3 \mathrm{D}$ reconstructed data sets:

Paired points matching (The most standard method): ISS localizer can be used for high accuracy image fusion ( 0.3 to $0.5 \mathrm{~mm}$ with CT/CT matching) .

Automatic fusion of same modalities (performed with the same patient position) (for instance T1 /T2 MRI exams): co-registration based on imaging system coordinates

Automatic fusion of framebased exams: co-registration based on frame coordinates Fusion of framebased/frameless exams: co-registration based on robot basis coordinates

Local Matching (in case of image distortions): local paired points or manual matching.
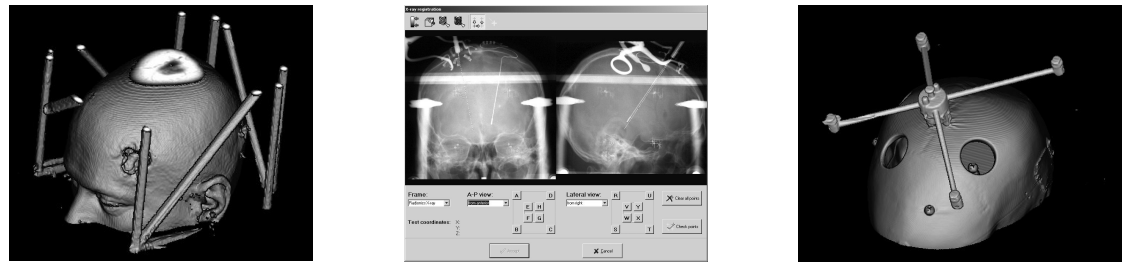

Fig. 1. Framebased 3D exam - Framebased 2D exams - Frameless 3D exam

\subsection{Co-registration}

\section{D/3D Co-registration}

To co-register two perpendicular X-rays views (framebased), the frame coordinates are used if the $3 \mathrm{D}$ exam is performed in framebased mode (automatic co-registration), and the robot basis if the $3 \mathrm{D}$ exam is performed in frameless mode.

\section{Integration of statistical data [1][6][7]}

Statistical data does not bring a high level of accuracy but they are very useful for target presetting and for trajectory verification. We are currently gathering our functional cases in a statistical database. Each target is validated by microelectrode recording and clinical results evaluation. In order to reduce the effect of patient to patient variations, statistical coordinates of functional targets are defined in an anatomical referential defined by anatomical landmarks (the anterior and posterior commissures and the mid-plane). This orthogonal referential is scaled with the thalamus height and the intercomissure distance. To preset functional targets with their statistical coordinates on images, we co-register the image referential with the described natomical referential. The landmarks are defined on images (2D or 3D data sets) and are introduced in a paired points matching algorithm. The same co-registration technique can also be applied for statistical brain atlases integration (Scaling can be performed by using the Talairach proportional grid). 


\section{Conclusions}

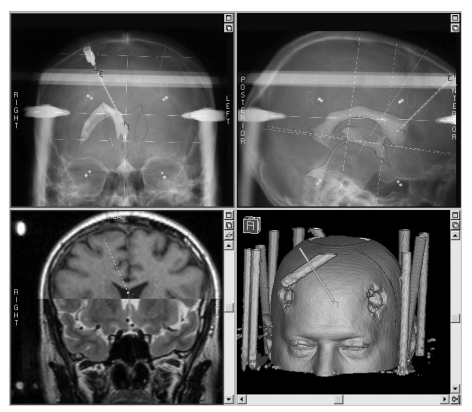

Some very simple, reliable, and useful methods are presented and discussed for integration of different modalities in the functional planning. These methods have been implemented in the VoXim ${ }^{\mathrm{TM}}$ software and are currently clinically used. They are helping us to define the optimal planning. Thanks to the NeuroMate ${ }^{\mathrm{TM}}$ stereotactic robot, the optimal targeting of the planned trajectory can be performed. This image and robotic guidance simplify dramatically the surgical procedure and leads to a very high level of accuracy.

\section{References}

1.Benabid, A.L., Pollak, P., Hoffmann D., Limousin P., Gao, D.M., LeBas, J.F., Benazzouz, A., Segebarth, C., Grand, S.

"Chronic Stimulation for Parkinson's Disease and other Movment Disorders"Textbook of Stereotactic and Functional NeuroSurgery Gildenberg-Tasker Mc Graw Hill 1998, p. 337349

2. Zamorano, L., Pandya, A., Li, Q.H., Perez-de-laTorre, R., Pittet, P. Badano, F., Robert, V "The clinical use and accuracy of the NeuroMate Robot for open NeuroSurgery"

Proceedings of the $14^{\text {th }}$ International Congress and Exhibition Computer Assisted Radiology and Surgery (CARS 2000) Elsevier Science B.V.p. 185-190

3. Eldridge, P., Byrne, P., Golash, A., Varma, T., Pittet, P. Badano, F., Nahum, B, Michel, JP

"The clinical use and accuracy of the NeuroMate Robot for open NeuroSurgery"

Proceedings of the $14^{\text {th }}$ International Congress and Exhibition Computer Assisted Radiology and Surgery (CARS 2000) Elsevier Science B.V. p. 980

4. Mösges, R., Lavallée, S.

"Multimodal information for Computer Integrated Surgery"

Computer Integrated Surgery Technology and Clinical Applications, The MIT Press 1996

5. Bucholtz, R.D., Sturm, C.D., Hogan, R.E.,

"The Use of Three Dimensional Images in Stereotactic Neurosuregry"

Textbook of Stereotactic and Functional NeuroSurgery Gildenberg-Tasker Mc Graw Hill 1998, p. 337-349

6. Coffey, R.J.

"Stereotactic Atlases in Printed Format"

Textbook of Stereotactic and Functional NeuroSurgery Gildenberg-Tasker Mc Graw Hill 1998, p. 237-248

7. Finnis, K.W., Starreveld, Y.P., Parrent, A.G., Sadikot, A.F., Peters, T.M.

"3D Functional Database of Subcortical Structures for Surgical Guidance in Image Guided Stereotactic Neurosurgery" Proceedings of the $2^{\text {nd }}$ International Conference Medical Image Computing and Computer-Assisted Intervention -MICCAI'99. Springer p. 758757 